\title{
Entretiens de recherche en psychopathologie: une approche par les mouvements de genres
}

\author{
Marie Carcassonne \\ Université Paris-Dauphine \\ marie.carcassonne@dauphine.fr
}

Pour éclairer les processus de co-construction du sens dans des entretiens de recherche en psychopathologie, nous allons appréhender ces entretiens à partir de la question des mouvements de genre qui les traversent. Nous présenterons d'abord les différences entre les notions de « macro-genre » et " micro-genre ». Puis nous observerons dans la dynamique de l'interaction, à partir d'extraits, la façon dont les interviewers proposent certains micro-genres et comment ceux-ci sont repris ou au contraire modifiés par les interviewés.

Les entretiens pris en compte ont été recueillis par des membres du Groupe de Recherche et d'Études sur les Conduites Ordaliques (GRECO), exerçant tous le métier de psychiatre ou de psychanalyste. Ces derniers avaient pour but d'explorer l'hypothèse psychopathologique de la « conduite ordalique ", définie ainsi: "répétition d'une épreuve comportant un risque mortel, dans laquelle le sujet s'engage afin, par sa survie, de prouver sa valeur intrinsèque, ainsi reconnu par les puissances transcendantes du Destin » (Charles-Nicolas \& Valleur 1982 : 90).

Les interviewers avaient réfléchi à l'avance à leurs questions de recherche et mis au point une grille ${ }^{1}$ simplifiée de thèmes à aborder en élaborant des " indices ordaliques » à rechercher au cours des entretiens : risque activement

1. Cette grille est consultable sur internet dans le rapport GRECO (Valleur 1994: 75). Notons qu'elle ne tombe pas dans le travers dénoncé par Lahire (1998 : 27) d'un questionnement purement abstrait concernant les interprétations de ses propres conduites. 
recherché et vécu positivement, prise de risque dans différents secteurs, autres « conduites associées " participant au versant ordalique (en particulier délinquance et prostitution, psychotraumatismes et ruptures précoces, vécu de ces événements). Les interviewés, tous volontaires, avaient été informés préalablement à l'entretien par oral et par écrit (lettre) du projet consistant à réfléchir sur leurs « récits de vie » recueillis à partir " d'interviews » enregistrées. Cinq des entretiens recueillis par le GRECO vont être ${ }^{2}$ ici présentés: trois toxicomanes (M., G., C. interviewés respectivement par P. pour les deux premiers et $\mathrm{Ch}$. pour le troisième), un délinquant prenant des risques au volant ( $\mathrm{R}$. interviewé par $\mathrm{Z}$.) et une anorexique devenue boulimique (F., interviewée par Y.).

\section{La notion de genre}

Dans toute interaction, l'activité (verbale ou non) qui y prend place est un des éléments majeurs façonnant la dynamique des énoncés produits. Ainsi, dans notre corpus, interviewers et interviewés peuvent être considérés comme les membres d'une équipe qui coopèrent (plus ou moins facilement, on le verra) pour accomplir une activité sociale pré-cadrée (Goffman 1991) de recherche de compréhension d'un comportement (addictif et/ou ordalique). De façon proche, Bakhtine-Voloshinov ${ }^{3}$ a bien décrit comment chaque « sphère » de l'activité humaine « (...) comporte un répertoire de genres de discours qui va se différenciant et s'amplifiant au fur et à mesure que se développe et se complexifie la sphère donnée " (Bakhtine 1984 : 265). Les " genres » de discours correspondent à des types relativement stables d'énoncés en tant qu'ils sont liés à une pratique sociale et/ou une activité. Ils sont issus de modèles historiquement constitués ${ }^{4}$ qui « orientent " (Schneuwly 1994 : 160) nos processus de réception et surtout de production des discours. Sinon, "l'échange verbal serait quasiment impossible " (Bakhtine 1984 : 285). En même temps, un genre n'est jamais répété ou imité, mais toujours refaçonné par le contexte local, rejoué (Goffman 1973) et donc modifié à chaque nouvelle interaction. C'est aussi en sens qu'Auer (1992) développe l'idée qu'un contexte est toujours en partie apporté et en partie construit localement. Un genre se caractérise, de ce fait, tout autant par des

2. Choisis parmi une quinzaine d'entretiens longs (env. 1,5 h.) sans principe spécifique.

3. Il est maintenant établi que la plupart des idées contenues dans certains ouvrages publiés sous le nom de Bakhtine reviennent en fait à Voloshinov. Pour la commodité du renvoi à la bibliographie, nous citerons désormais uniquement Bakhtine.

4. Schütz (1987) décrit lui aussi les activités (pas seulement discursives) comme s'appuyant sur des "ressources typifiantes ", et les considère sous deux traits fondamentaux: non seulement leur historicité, mais aussi leur caractère collectif et intersubjectif. 
aspects stabilisés que par un style individuel de mise en œuvre. Clot (1999) a souligné en développant la notion de " genre d'activité » que ce sont ces aspects stylistiques qui, lorsqu'ils sont repris par le plus grand nombre, font évoluer les macro-genres.

\subsection{Macro-genre et micro-genre}

Nous proposons d'appeler « macro-genre » la liaison entre discours et activité ou pratique sociale, en tant que norme abstraite attendue ou "représentation »: genre entretien, genre exposé, etc.; " micro-genres » les types d'organisations discursives plus locales se repérant concrètement dans le déroulement d'un discours. Kerbrat-Orecchioni (2003) fait une distinction similaire et souligne qu'un macro-genre (qu'elle nomme G1) s'identifie par rapport à un externe au discours (la situation, l'activité) tandis que le micro-genre (qu'elle nomme G2) s'identifie par rapport à des critères internes au discours. Pour Adam (1992) comme pour Bronckart (1997), c'est aussi à ce niveau des micro-genres (qu'ils nomment différemment et en procédant différemment), et non des macro-genres, que peut s'établir une typologie des discours, à partir du repérage d'usages différenciés de certains modes énonciatifs. Soulignons toutefois que dans une interaction orale, les frontières entre micro-genres ne sont jamais nettes ${ }^{5}$ : ils peuvent s'enchaîner dans le déroulement concret de l'interaction par alternance (par ex. une explication puis un récit), ou être mélangés (par ex. un récit explicatif). Ainsi nous avons analysé en comparant différents types d'entretiens de recherche la difficulté qu'il pouvait y avoir à établir des frontières entre les micro-genres récit, explication, justification, argumentation et interprétation (Carcassonne et alii 2001). Lapparition de tel ou tel microgenre dans tel macro-genre peut correspondre aux attendus normés liés à ce macro-genre ou au contraire les bousculer, et donner parfois lieu alors à une " négociation du genre " (Kerbrat-Orecchioni 2003 : 9). Quand un micro-genre apparaît de façon très locale et ne renverse pas les attentes liées au macro-genre, Vion (1995) propose de l'isoler comme un « module » à part: ainsi un « module conversationnel » peut-il surgir dans le déroulement du macro-genre entretien.

\subsection{Quel macro-genre pour le corpus?}

Dans le cas de notre corpus, les questions de recherche sont connues préalablement par chacun des interactants mais donnent nécessairement

5. Comme l'ont souligné entre autres Lacoste 1986; Boutet 1989; Vincent 1994; cf. Carcassonne 1997 : 202-204 pour un résumé de ces différents apports. 
lieu à des représentations différentes: elles ont contribué à une forme de "pré-occupation » (Clot, 1999) des « places discursives » (Flahaut 1978 Vion 1995). Certains mouvements de places montrent en effet que les interviewers, qui exercent tous le métier de psychiatre ou psychanalyste, sont aussi " préoccupés " par des projets thérapeutiques. Blanchet a défini cette position comme étant celle de l'entretien clinique en soulignant qu'elle était « intenable » $(1985$ : 9) : car pour lui l'objectif d'un entretien de recherche est la construction d'une connaissance d'un problème (même subjectif) et l'élaboration d'un savoir socialement communicable; tandis que l'objectif d'un entretien thérapeutique est la constitution d'un savoir privé peu communicable, grâce au jeu de relations imaginaires envers le thérapeute (transfert). Mais cela n'est possible selon Blanchet que si le patient a la garantie fondamentale que son discours ne sera pas objet de connaissance; or l'entretien clinique a la prétention de concilier les deux approches.

Les entretiens présentés ici se situent toutefois davantage du côté de l'entretien de recherche et s'annoncent comme tels; mais la dénivellation du macro-genre « entretien de recherche » au macro-genre " entretien thérapeutique ", se repère néanmoins plusieurs fois. Cette ambiguiité du statut des interactants et par là même du " macro-genre " ${ }^{7}$ de l'interaction donne parfois lieu à une véritable négociation entre les interactants à propos du macro-genre qu'ils estiment mobiliser. Cette négociation peut s'observer très concrètement à partir des mouvements de places, comme le montre l'exemple suivant, où, au milieu d'un entretien, l'interviewé (M., toxicomane) évoque son frère plus jeune:

Exemple $n^{\circ} 1^{8}$

M: lui il est parti aux États-Unis aussi voilà il est revenu et moi je je prenais de la came j'étais complètement dedans et alors pff ça c'est ébranlant quoi! Son frère qui était pour lui euh Staline tu vois un peu comme pour les Russes

$P$ : Staline?

$\mathrm{M}$ : non mais pour moi c'est l'image à la con hein tu vois le dur le totem

6. Flahaut a posé le concept de rapports de places, en insistant sur le fait que toute parole convoque l'interlocuteur à une place corrélative. Pour Flahaut, la distribution des places est tributaire du cadre dans laquelle elle se déploie, et plus largement du contexte social et culturel.

7. L'affirmation de Goffman selon laquelle les différents participants s'accordent en général sur les définitions de la situation ne se vérifie donc pas ici.

8. Les conventions de transcription sont en annexe. 
À la fin de l'entretien, alors que l'interviewé indique qu'il a souffert de l'absence de son père, l'interviewer reprend à distance 9 et en partie les propos de $M$. en les recadrant différemment:

$\mathrm{P}$ : euh cette histoire du père tu en avais parlé de Staline?

M: (il rit) Il me viole! Mais vous faites quoi au fait? Psychanalyste? Psychologue?

$\mathrm{P}$ : mais là dans cet entretien je suis oui... je suis de formation psychanalytique mais... c'est évident que cet entretien a des orientations... euh une orientation comme ça mais là on parle de... euh de... nous sommes à ça par ailleurs il y a notre pêche dans la suite de ta cure de ta postcure de demander à l'équipe de $\mathrm{X}$ d'aller voir si y a pas un psychothérapeute que tu pourrais aller voir c'est à toi de voir

Outre qu'il accuse son interviewer de le violer, $\mathrm{M}$. le désigne à la troisième personne et passe au vouvoiement: cette prise de distance discursive et cette violence dénoncée par l'interviewé sont certainement liées au fait que l'interviewer s'était annoncé comme chercheur mais occupe finalement ici une place d'interprète-analyste " psy ». Son statut, en principe " externe » à l'entretien, de thérapeute " psy » et le rôle qui lui est lié (aider l'autre à mieux se comprendre) télescope ici le rôle annoncé initialement de chercheur en psychopathologie. Les places discursives éclairent donc la façon dont les uns et les autres se représentent leur(s) rôle(s) dans l'entretien, et renseignent par là même sur la conception que les interviewers comme les interviewés se font de la recherche à laquelle ils prennent part: de recherche avec une coloration thérapeutique pour les interviewers, surtout de recherche pour les interviewés ${ }^{10}$.

\section{Des micro-genres non induits par les interviewers}

Dans un article antérieur (Carcassonne 2006) nous considérons l'activité narrative se développant dans ces entretiens comme une co-construction par reformulations, questions et mouvements successifs. À partir d'extraits des mêmes entretiens, nous pointons l'aspect explicatif des micro-genres récits, en distinguant précisément: le récit explicatif ou l'explication narrativisée offerte, et parmi les explications incluses dans ces micro-genres récits, celles qui sont proposées par l'enquêté après avoir été induites par

9. Lorsque un contenu non repris à proximité l'est à distance, cela en dit plus que le contenu en question. Grunig \& Grunig (1985 : 244), s'inspirant du nom d'une rivière des Causses, région où les cours d'eau disparaissent sous terre puis resurgissent, ont proposé d'appeler "Dourbie" ce phénomène qui consiste à revenir sur un sujet déjà abordé préalablement puis abandonné.

10. Faute de place, nous ne pouvons joindre les extraits montrant cet aspect. 
l'interviewer, proposées par l'interviewer et refusées par l'enquêté, demandées par l'interviewer, puis fournies ou non, par l'enquêté. Nous soulignons en outre le fait que l'interviewer introduit ou revient souvent sur certains thèmes qui ne sont pas toujours ceux que l'interviewé avait choisi de développer spontanément ${ }^{11}$. Les mouvements des interviewers apparaissent donc comme orientant les propos des interviewés, en particulier au niveau de l'interprétation des événements de vie.

La notion de " mouvement » (François 1984 ; 1989a) renvoie à un double mode interprétatif: un mode où le récepteur établit des liens entre les énoncés co-produits in praesentia par les interlocuteurs en situation (aspect fermé de la production) ; un mode, incluant le précédent, où le récepteur (qu'il soit membre de l'interaction ou analyste, comme nous, de cette même interaction) établit des liens entre le discours et des éléments externes au discours (aspect ouvert de la réception).

Dans ce cadre, trois niveaux de significations, se recouvrant progressivement, peuvent être décrits (François, 1989b). Ces niveaux sont liés: aux éléments de contenu localisables ponctuellement et précisément; aux enchaînements entre énoncés, localisables dans le déroulement discursif; aux significations dessinées dans le déroulement du discours. Ce dernier niveau, qui inclut les précédents, dépend « de ce qu'on rapproche, dans le discours de celui qui parle ou dans le sens qui se dessine par comparaison avec ce qu'on attend ou avec le discours des autres " (François 1993 : 34). Une des significations dessinées que nous évoquerons réside dans la notion de style (François 1993; Salazar 1999) qui permet de pointer, même à l'oral, la spécificité du résultat de la mise en mots, en tant que façon de dire (style énonciatif) et d'interagir avec l'autre (style interactif).

Dans ce cadre interprétatif, nous allons justement décrire le style des micro-genres circulant dans les entretiens en insistant ici sur certains enchấnements de micro-genres: tandis que certaines questions des interviewers proposent certains micro-genres (par exemple demande de récit factuel, demande de récit interprétatif), les réponses des interviewés gardent souvent le thème induit par la question mais pas le micro-genre proposé. C'est-à-dire qu'au-delà des orientations induites par les interviewers, les réponses des interviewés peuvent modifier les micro-genres proposés, sans que l'on assiste pourtant à une rupture entre les questions et les réponses. Ces décalages ne sont donc pas thématisés, mais repérables au niveau des enchaînements, dans les différences entre les micro-genres proposés par les interviewers et ceux proposés les interviewés. Si les micro-genres sont

11. Voir note 9. 
actualisés dans l'entretien au cours d'une co-production, il s'agit cependant d'une co-production qui contrarie parfois les propositions des interviewers, et ces contrariétés vont justement être l'un des objets de notre analyse.

Les différences interactives et énonciatives du style des micro-genres dominants ${ }^{12}$ dans chaque entretien vont apparaître en outre redoublées par la réception de l'entretien dans son ensemble (en tant que macro-genre), en particulier au niveau de la mise en mots de la temporalité: cyclique, répétitive, passive pour quatre d'entre eux; structurée par un avant/après pour F., avec davantage de contenus concernant des projections dans le futur, mais aussi de codages de soi en termes actifs et de procédés linguistiques de distanciation.

\subsection{Dominance du micro-genre "récit factuel " chez C. et $R$.}

C. et $\mathrm{R}$. se racontent par le biais d'un micro-genre " récit factuel » homogène tout au long de l'entretien, quels que soient le thème des questions et le moment de l'entretien. Ils maintiennent ce micro-genre même lorsque les questions des interviewers montrent que ces derniers attendent un microgenre " récit " plus interprétatif. Aux questions induisant un micro-genre interprétation psychologique, ils répondent toujours dans un micro-genre " récit factuel » caractérisé par la dominance de la catégorie des verbes d'action ainsi que par une succession de phrases courtes créant un effet de rapidité et renforçant l'absence d'élaboration et d'interprétation des faits évoqués.

$\mathrm{R}$. produit tout au long de l'entretien des micro-récits, par exemple pour renvoyer à sa dépendance au vol de voitures ("c'est ma petite drogue à moi les voitures ", dit-il à un moment). S'il évoque la prison, lieu d'où il parle au moment où il est interrogé, c'est toujours pour envisager le moment où il pourra sortir et voler à nouveau des voitures. La doxa qui consisterait à blâmer ce comportement n'est jamais thématisée, au contraire: «c'est mon fort les voitures ", dit-il à un moment. Il n'essaye jamais d'expliquer ni d'interpréter ses conduites, même lorsque l'interviewer le demande très explicitement.

Exemple $\mathrm{n}^{\circ} 2$

$\mathrm{Z}$ : dès qu'une voiture vous plaît euh... [vous la volez?]

$\mathrm{R}:$

(a) ah même si je vois quelqu'un dedans hein! c'est pareil (...)

(b) je suis le gars quand il s'arrête je lui tire sa caisse ah ouais

(c) si si y a une voiture qui me plaît c'est ça me fait tilt dans la tête c'est fini

12. Notons que la notion de dominance relève nécessairement ici d'un aspect « subjectif " lié au mouvement interprétatif du récepteur. 
(d) même les les Espaces là les petits camions quoi je veux dire c'est pareil quoi

(e) faut que ça me tape dans la tête

(f) le matin je me réveille je la trouve plus belle je vais en chercher une autre parce que $j$ 'aime plus celle-ci

(g) il me faut une voiture que j'aime le jour qui vient

Lorsque l'interviewer lui demande explicitement vers la fin de l'entretien une interprétation de cette temporalité cyclique dans laquelle il vit, $\mathrm{R}$. répond à nouveau en énonçant des faits:

Exemple $n^{\circ} 3$

$\mathrm{Z}$ : tu ne t'es pas demandé ce que ça veut dire de venir si souvent en prison? $\mathrm{R}$ : ben disons que je savais que j'allais finir comme ça pff je prenais trop de risques mais euh on m'a jamais attrapé en flagrant délit toujours les keufs i sont venus me chercher comme ça pour un contrôle d'identité pour des conneries

De façon proche, $\mathrm{C}$. répond comme $\mathrm{R}$. aux questions de l'interviewer, mais avec une différence de micro-genre telle que ce qu'elle dit n'a finalement plus aucun rapport avec les questions qu'on lui pose même s'il y a accord au niveau référentiel. Ses réponses faisant suite à des demandes d'actualisation de faits intérieurs sont soit minimales, soit déplacées vers un discours événementiel et objectif où domine l'action. Le thème suggéré par la question est alors conservé mais pas le micro-genre ni la catégorie.

Exemple $n^{\circ} 4$

$\mathrm{Ch}$ : et tu te souviens de ce que tu éprouvais dans la situation [de cambriolages] ? comment:::comment tu vivais ce type de:::de situation?

$\mathrm{C}$ : c'que j'éprouvais en tout cas je pas d'peur pas d'crainte ça va tellement vite! puis on pense à l'argent qu'y a parce que:::: on se::::on s'doutait de c'qu'y'avait à peu près y'avait douze bâtons!

Ch: la peur y en avait pas avant y en avait pas sur le moment y en avait après?

C: un petit peu avant peut être après non! après on est trop content on speed parce que faut se sauver vite faut pas perdre de temps! mais euh on pense pas à on pense pas::: comme vous dites quoi, on pense pas tout de suite!

Dans ce dernier énoncé, C. explicite clairement sa difficulté à entrer dans le micro-genre explication psychologique de son interlocuteur. Tous ses récits sont constitués d'une succession de phrases courtes où prédominent les événements et les verbes d'action, ce qui crée un effet de rapidité.

C. et R. n'entrent donc jamais dans le micro-genre interprétatif demandé par les interviewers et utilisent un micro-genre « récit factuel » quelles que soient les questions posées, avec les spécificités énonciatives et thématiques suivantes: un grand nombre de codages de soi en termes passifs; des 
descriptions d'un état psychologique par le biais d'images ou d'actions concrètes (exemple 2 en (c), (f), exemple 3, exemple 4); la thématisation d'une temporalité cyclique (exemple 2 en (g), exemple 3, exemple 4). $S$ 'ils se codent parfois en termes actifs, c'est pour relater des faits et des actions ponctuelles et répétitives, sans projection dans le futur, relatifs à leurs conduites addictives.

\subsection{Dominance du micro-genre récit explicatif-justificatif chez $M$. et $G$.}

M. et G. se racontent quant à eux de façon dominante dans des microgenres intermédiaires entre le récit et l'explication.

C'est le cas dans l'exemple 5 ci-dessous, où l'interviewer cherche à obtenir des informations purement factuelles tandis que $\mathrm{G}$. répond dans un micro-genre intermédiaire entre récit et explication. Après avoir fourni les informations factuelles demandées par P. (en 10G, 12G, 14Ga), G. continue à répondre sur le même objet que celui sur lequel on l'interroge (sa conduite addictive) mais en empruntant d'autres micro-genres: d'abord un micro-genre « explication paraphrastique » de (b) à (m) puis un microgenre « récit explicatif » de (n) à (o).

Cet exemple est en outre remarquable par la grande énergie que met $\mathrm{G}$. à déplacer le codage de soi en termes actifs proposé par l'interviewer (en $11 \mathrm{P})$ par un codage de soi en termes passifs :

Exemple $n^{\circ} 5$

$9 \mathrm{P}$ : là tu as quel âge?

10G: vingt-huit

$11 \mathrm{P}:$ et :::tu as t'as pris contact avec cet autre centre de... désintoxication?

12G: oui

$13 \mathrm{P}$ : et tu avais quel âge?

$14 \mathrm{G}$ :

(a) j'avais ::::24 ans

(b) enfin c'était plus un concours de circonstances dans le sens où j'habitais dans la même ville

(c) donc euh par rapport au fait que j'étais toxicomane

(d) qu'y avait dans la même ville un centre d'aide aux toxicomanes

(e) donc ça allait euh l'un découlait de l'autre quoi

(f) c'est pas une démarche que j'ai faite euh

(g) j'ai pas été voir un centre euh enfin c'était euh

(h) pour moi c'est quelque chose de de tout à fait

(i) en fin de compte ça s'inscrivait pas dans une réelle démarche

(j) c'était plus pour compléter enfin pour euh pour être cohé cohérent quoi

(k) et puis pour pas me sentir isolé dans ma toxicomanie 
(m) donc je voulais prendre je voulais savoir un peu comment ça se passait

(n) parce que jusqu'à enfin jusqu'à ce moment-là j'avais toujours soit essayé de décrocher par mes propres moyens par rapport aux informations que j'avais par rapport au traitement

(o) bon c'était pas très concluant

G. lève ici un éventuel malentendu (de façon consciente ou non) qui aurait pu s'installer à la suite de sa réponse 12 (oui) en déplaçant le thème proposé par P. (les données factuelles des prises en charge passées) pour thématiser justement l'absence de réelle volonté de sa part pour être pris en charge; ce faisant, il sort progressivement du micro-genre « chronologie factuelle " proposé par P. pour entrer dans un micro-genre " explication paraphrastique » de la passivité dans laquelle il vit.

Ce thème de la passivité est renforcé par le mode énonciatif actualisant ce micro-genre, caractérisé par un mouvement de désancrage énonciatif (par rapport à la question 11P.).

Ce désancrage est opéré en (b) par l'utilisation d'actualisateurs indéterminés (cétait, repris ensuite en (e) ça, et en (f) c'est), du pronom indéfini un, et de l'élément nominal concours de circonstances.

L'énoncé (e) thématise comme l'énoncé (b) une causalité externe, ce qui est renforcé par le mode énonciatif désancré.

Dans les deux énoncés suivants (f) et $(\mathrm{g})$, le « je » impliqué par la question 11P est explicitement refusé.

En (i), G. synthétise son refus du mode agentif impliqué par la question 11P, et cela sur le même mode énonciatif que dans l'énoncé (b) (actualisateur indéfini ça, pronom indéfini une, nominalisation démarche).

En (j), le désancrage énonciatif est maintenu avec l'utilisation de l'infinitif être cohérent, même s'il est moins fort qu'avec la nominalisation précédente.

Un mouvement d'ancrage énonciatif est finalement opéré en $(\mathrm{k})$ avec les pronoms « me » et " ma ", mais toujours sur mode passif (me sentir).

Une intentionnalité de l'événement raconté apparaît ensuite en (m) mais G. se présente alors sur le mode d'un personnage très distancié par rapport à la notion de prise en charge de son addiction (je voulais savoir un peu comment ça se passait).

De (n) à (o), le mouvement d'ancrage énonciatif se poursuit, mais $G$, en passant d'un micro-genre " explication paraphrastique " à un micro-genre " récit explicatif " se code alors comme l'agent d'actions non abouties, commentées négativement (javais essayé, c'était pas très concluant).

G. tente donc de façon dominante d'expliquer-interpréter ses conduites en utilisant spontanément un micro-genre récit « explicatif justificatif », et 
cela alors même que les enchaînements de l'interviewer montrent que ce micro-genre n'est pas celui qu'il attend.

M. se raconte dans un micro-genre très proche de celui de G. Ainsi lorsqu'il est interrogé sur la manière dont il envisage son destin, il répond en proposant une « explication psychologique » de son absence de motivation. Le micro-genre qu'il propose est par contre cette fois-ci en accord avec celui que propose l'interviewer dans sa question.

Exemple $n^{\circ} 6$

$\mathrm{P}$ : est-ce que tu crois que c'est ton destin d'être drogué

$M(\ldots)$

(a) mais j'ai pas encore eu euh la volonté ou

(b) j'sais pas c'qui me faut pour arriver à faire ces démarches-là

(c) c'est c'est c'est c'qu'on appelle la motivation

(d) moi je suis un mec qui est démotivé

(e) je suis démotivé

En (d) et (e), M. se code comme le siège d'une propriété négative intrinsèque car non bornée temporellement. Au niveau des significations dessinées, ces énoncés s'associent aux énoncés (a) et (b) où $M$. se code en termes passifs pour produire l'effet d'une présentation de soi dévalorisée et sans projet.

Il peut arriver à d'autres moments de l'entretien que les micro-genres utilisés par $\mathrm{M}$. et $\mathrm{G}$. varient en fonction du thème évoqué, certains thèmes entraînant même parfois des micro-genres factuels s'apparentant alors à des conduites de dénégation (Carcassonne \& Valleur 1997). Mais cette conduite discursive reste alors secondaire par rapport au style énonciatif du micro-genre dominant récit " explicatif » ou " justificatif » (de la passivité et de l'absence de projet) tel qu'il a été décrit dans les exemples 5 et 6 .

Par rapport à C. et R., M. et G. entrent donc davantage dans le microgenre interprétatif demandé par les interviewers, avec un style interactif différent car moins systématique: ils peuvent fournir un micro-genre demandé (exemple 6), refuser de le faire, ou en fournir un non-demandé (exemple 5). Dans tous les cas, le micro-genre dominant qu'ils utilisent est celui du récit explicatif d'une absence de motivation. Il se caractérise au niveau du style énonciatif par un grand nombre de codages de soi en termes passifs, lesquels sont renforcés si la question est posée en termes actifs (exemple 5), et au niveau thématique par une absence de projections de soi dans le futur (exemples 5 et 6 ). 


\subsection{Dominance du micro-genre récit interprétatif chez $F$.}

Une toute autre dynamique apparait dans l'entretien de F. qui se raconte dans un micro-genre intermédiaire entre le récit et linterprétation, proche du discours " psy ", avec moins de codages de soi en termes passifs et davantage de procédés énonciatifs de distanciation. F. est aussi la seule des interviewés à donner une valeur de témoignage à son discours et à présenter son récit de vie comme structuré par un avant (la reprise de son travail) et un après, avec des projections de soi dans le futur. Ce thème, présent dans l'exemple 7 commenté ci-dessous, est repris plusieurs fois dans l'entretien.

Exemple $\mathrm{n}^{\circ} 7$

Y: Vous avez l'impression que c'est comme quelqu'un d'autre qui vous a donné le goût de manger?

F:

(a) oui j'leur en veux de ça de la façon dont i m’ont soignée

(b) et on m'a dit d'ailleurs les cures d'insuline on en fait plus hein! ça n'existe plus hein! c'est trop dangereux ils le font plus ça

(c) en fait souvent à XX les filles que j'ai vues qui étaient comme moi bon elles sont encore anorexiques elles hein et elles le resteront sans doute et avec des perfusions hein

(d) c'est sûr que c'est pas évident

(e) moi c'qui m'embête c'est que je me bousille l'estomac

(f) et puis toute ma vie je vomirai facilement hein ça je le sais pff c'est un problème quoi

(g) mais euh c'qui est bien par contre c'est que bon l'invalidité ça m'a beaucoup aidée financièrement

(h) et ce qui m'a aidée c'est qu'ils m'ont proposé un stage de remise à niveau

(i) je trouve qu'ils devraient faire pour les gens qui sont au chômage un jeune au chômage

(j) qui peut faire un stage même s'il est mal payé si c'est un gars qui a envie de faire quelque chose

(k) ben c'est vachement important hein moi je favorise le les gens tous les stages qui sont proposés

(l) parce que vous savez moi c'est ça qui m'a sortie

(m) j'avais un but j'savais que le matin à huit heures il fallait que je sois là que $j$ 'aie quelque chose à faire

(n) j'ai fait mon CV j'ai refait un CV j'ai appris des choses bon ben c'était

(o) l'essentiel pour moi hein ça m’a aidée à m'en sortir un peu

Dans cet extrait, F. commence par critiquer la façon dont on elle a été soigné (a à d). En même temps, elle ménage la face de son interlocuteur:

- en faisant appel à une source énonciative indéterminée: le pronom " on " (b: on m'a dit) lui permet de renvoyer indifféremment au corps médical (et de se placer ainsi sur un terrain d'un savoir partagé avec son 
interlocuteur) ou à d'autres malades pour indiquer qu'elle est au courant de l'arrêt des modes de traitement qu'elle a subis et qu'elle critique;

- en utilisant une tournure impersonnelle en (d: c'est pas évident) qui permet elle aussi de fondre son propre point de vue à celui des médecins et de formuler des reproches au monde médical tout en créant une connivence avec son interlocuteur.

En (e, j), elle évoque les problèmes liés à son cas personnel et singulier puis opère un déplacement vers le générique ( $\mathrm{i}$ à $\mathrm{k}$ ) ; une prise de parole collective, d'appartenance à un groupe, apparaît en (i), introduite par le verbe d'opinion « je trouve " qui implique entre autre « un jugement personnel fondé sur l'expérience " ainsi qu' " une expérience de la chose elle-même " (Ducrot 1980). En effet, c'est en fonction de sa propre expérience, évoquée déjà à distance ${ }^{13}$, et reprise ici en $(1, \mathrm{~m}, \mathrm{n}, \mathrm{o})$, que $\mathrm{F}$. se permet de porter un jugement plus collectif. Elle revient en $(\mathrm{m}, \mathrm{n})$ sur son cas personnel en se codant en termes actifs et en se projetant dans le futur, ce qui entre en contraste avec la façon de se dire des autres interviewés (cf. les soulignés des exemples précédents).

Le type de mouvement énonciatif présent ici, comportant une justification du générique par une expérience particulière se retrouve à propos de nombreux autres thèmes au cours de l'entretien, avec une dominance pour celui qui concerne l'explication de la cause de la maladie et de son traitement. Par rapport à ce thème, F. prend la place discursive d'une quasicollègue et cherche à aider son interlocuteur à mieux soigner les autres. La capacité dont fait preuve F., à la différence des autres interviewés, à convoquer différentes sources énonciatives et à se mettre à la place de son interlocuteur, a été pointée comme efficace pour mettre à distance la douleur. Être capable de se mettre à la place de l'autre - l'interlocuteur - au plan interlocutif comme au plan de l'intersubjectivité semblerait aller de pair « (...) avec la capacité - du moins telle qu'elle est manifestée dans le discours - de prendre du recul face à l'épreuve ou à la souffrance ", (Vion et alii 2006 : 212). F. fait de ce point de vue preuve d'une capacité réflexive sur le discours produit en utilisant un micro-genre très interprétatif allant parfois même au-delà des attentes des interviewers et que nous allons, en conclusion de cette partie, caractériser par comparaison avec le style des micro-genres dominants des autres entretiens.

F. répond aux demandes d'interprétation des interviewers, fournit des interprétations de ses conduites même quand les interviewers n'en demandent pas. Le micro-genre dominant récit interprétatif de soi qu'elle propose

13. Voir note 10. 
se caractérise au niveau du style énonciatif par l'utilisation de sources énonciatives variées et des codages de soi en termes actifs pour évoquer le futur, phénomènes que l'on ne retrouve pas chez les autres interviewés; par la mise en mots une temporalité structurée par un avant/après qui contraste avec la temporalité cyclique, répétitive et sans projet mise en mots par les autres interviewés ${ }^{14}$.

\section{Conclusion}

L'analyse de certains enchaînements nous a permis de constater que les interventions des interviewers orientent pour une part le discours des interviewés: elles privilégient selon les moments de l'entretien (et en fonction de la grille préétablie) des questions factuelles ou des questions interprétatives; ces interventions proposent en outre parfois de façon ambiguë des considérations thérapeutiques qui ne sont pas toujours acceptées par les interviewés.

Au-delà de ces orientations induites par les interviewers, les interviewés imposent cependant des styles de micro-genres préférentiels pour répondre aux questions: au niveau du style interactif, dans la façon de déplacer, chacun à leur manière, les micro-genres demandés; au niveau du style énonciatif, dans la façon d'utiliser des codages de soi en termes passifs ou actifs, tournés vers le passé ou vers le futur, et de prendre en compte ou non le point de vue de l'interlocuteur.

Ces différences permettent de caractériser le discours de chaque interviewé par un style de micro-genre dominant et dessinent sur l'ensemble des entretiens des significations encore plus fortes que celles qui apparaissent au niveau des seuls enchaînements locaux.

Ainsi, le style énonciatif toujours très factuel de C. et R. contrarie les attentes de l'interviewer par rapport au micro-genre demandé; le style énonciatif explicatif-justificatif de M. et G. correspond plus souvent aux attentes des interviewers; tandis que le style énonciatif très interprétatif de $F$. va souvent au-delà des attentes de l'interviewer en termes de micro-genre.

S'ils présentent une pathologie de la dépendance, les interviewés manifestent finalement une indépendance ${ }^{15}$ dans leur capacité à introduire les micro-genres par lesquels ils répondent aux interviewers.

14. La question de savoir si la différence de style des micro-genres dominants dans le discours de F. par rapport aux autres interviewés est liée à une disposition personnelle, éventuellement travaillée par la psychothérapie, et/ou à l'aspect moins ordalique et moins addictif de sa pathologie, reste entière.

15. Guilhaumou (2002) a pointé ce même fait sur une population proche. 


\section{Références citées}

Adam J.-M. (1992), Les textes: types et prototypes, Paris, Nathan.

Auer P. (1992). Introduction: John Gumperz's Approach to Contextualization (p. 1-37). In P. Auer \& A. di Luzio (eds). The contextualization of language. Amstersdam: Benjamins.

Bakhtine M. (1984), Les genres de discours, dans Esthétique de la création verbale, Paris, Gallimard (p. 265-308).

Blanchet A. (1985), Interviewer, dans A. Blanchet, M. Ghiglione \& J. Massonat (dirs), Les techniques d'enquêtes en sciences sociales, Paris, Dunod, (p. 82-126).

Boutet J. (1989), Construire le sens, Neuchâtel, Peter Lang.

Bronckart J.-P. (1997), Activité langagière, texte et discours. Pour un interactionnisme socio-discursif, Paris, Delachaux et Niestlé.

Carcassonne M. (1997), Énonciation, mouvements et temporalités dans trois récits de vie recueillis en interaction, Thèse de doctorat de linguistique, Université René Descartes-Paris V.

- (2006), Présentation de soi dans l'entretien clinique: narration, explication, interprétation, dans M. Grossen \& A. Salazar-Orvig (dirs.), L'entretien clinique en question: analyse des interactions verbales d'un genre communicatif hétérogène, Paris, Belin (p. 213-231).

Carcassonne M. \& Valleur M. (1997), Discours et conduites addictives, dans A. Blanchet (éd.), Champs de recherche en psychologie clinique, Paris, Dunod (p. 69-95).

Carcassonne M., Salazar-Orvig A. \& Bensalah A. (2001), Des récits dans des entretiens de recherche: entre narration et interprétation, dans J. Bres \& D. Vincent (coord.) Le récit oral conversationnel, Revue québécoise de linguistique, 29-1, p. 97-122.

Charles-Nicolas A. \& Valleur M. (1982), Les conduites ordaliques, dans C. Olievenstein, La vie du toxicomane, Séminaire de l'hôpital Mamottan, Paris, PUF (p. 82-99).

Clot Y. (1999), La fonction psychologique du travail, Paris, PUF.

Ducrot O. (1980), Je trouve que, dans Les mots du discours, Paris, Éditions de Minuit (p. 57-91). 
Flahaut F. (1978), La parole intermédiaire, Paris, Le Seuil.

François F. (1984), Interprétation linguistique et psychopathologie, L'Évolution psychiatrique, 49-2, p. 415-450.

- (1989a), De quelques aspects du dialogue psychiatre-patient. Places, genres, mondes et compagnie, C.A.L.A.P., 5, p. 39-89.

- (1989b), Sémantique et signification, La linguistique, 25-1, p. 75114.

- (1993), Pratiques de l'oral, Paris, Nathan.

Goffman E. (1973), La mise en scène de la vie quotidienne, T.1: La présentation de soi, Paris, Minuit.

- (1991), Les cadres de l'expérience, Paris, Minuit.

Grunig B.N. \& Grunig R. (1985), La fuite du sens, La construction du sens dans l'interlocution, Paris, Hatier.

Guilhaumou J. (2002) Le corpus en analyse de discours: perspective historique, Corpus $n^{\circ} 1$ : corpus et recherches linguistiques, http://revel.unice. $\mathrm{fr} /$ corpus/personne.html?type=auteur\&id=73.

Kerbrat-Orecchioni C. (2003), Les genres de l'oral: Types d'interactions et types d'activités, Contributions à la journée organisée par Catherine KerbratOrecchioni et Véronique Traverso le 18 avril 2003, Université Lumière Lyon, http://icar.univ-lyon2.fr/Equipe1/actes/journees_genre.htm

Lacoste M. (1986), La narrativisation dans une situation d'interview, Études de linguistiques appliquées, 63, p. 50-62.

Lahire B. (1998), Logiques pratiques: le "faire" et le "dire sur le faire", Recherche et Formation, 27, p. 15-28.

Salazar-Orvig A., (1999), Les mouvements du discours; style, référence et dialogue dans des entretiens cliniques, Paris, L'Harmattan.

Schneuwly B. (1994), Genres et types de discours, dans Y. Reuter (éd), Les interactions lecture-écriture, Berne, Peter Lang (p. 155-174).

Schütz A. (1987), Le chercheur et le quotidien. Phénoménologie des sciences sociales, Paris, Méridiens Klincksieck.

Valleur M. (dir.), (1994), GRECO, Toxicomanie et mort, rapport de recherche, MESR, DGLT, Association Descartes, Paris; consultable sur http:// www.hopital-marmottan.fr/download/greco.pdf. 
Vincent D. (1994), La fonction des narrations dans les entrevues sociolinguistiques, dans J. BRES (dir.), Le récit oral, Montpellier, Presses de l'université de Montpellier.

Vion (1995), La gestion pluridimensionnelle du dialogue, Cahiers de linguistique française, 17b, Les différents plans d'organisation du dialogue et leurs interrelations: actes du Vième Colloque de pragmatique de Genève, 15-17 juin 1995, p. 179-203.

Vion R., Rouveyrol L., Maury-Rouen C., Bremond C., Boström E., Assef C. \& Noël-Jorand M.C. (2001), Outils linguistiques pour l'analyse linguistique des discours et des émotions, dans Revue française de psychiatrie et de psychologie médicale, 5-49, p. 49-56.

\section{NORMES DE TRANSCRIPTION :}

$10 \mathrm{G}$ : Le chiffre précédant $\mathrm{G}$ correspond à l'ordre de la prise de parole au cours de l'entretien : 10G indique donc que la dixième prise de parole au cours de l'entretien revient à $\mathrm{G}$; ces chiffres ne sont indiqués que lorsqu'ils permettent de lever toute confusion lors du renvoi aux exemples dans le commentaire analytique. 10G (a) : Les lettres entre parenthèses correspondent à un découpage (sans logique spécifique) de la transcription au sein d'une même prise de parole. Elles permettent de faciliter le renvoi à certaines expressions (par le biais d'une lettre) lors de l'analyse

:::: allongement de syllabe

(...) coupure dans la transcription jointe

? intonation montante

! intonation exclamative 
